\title{
Validating clinical characteristics of primary failure of eruption (PFE) associated with PTH1R variants
}

\author{
Cristina Grippaudo ${ }^{1,2}$, Isabella D'Apolito ', Concetta Cafiero ${ }^{3}$, Agnese Re ${ }^{4}$, Pietro Chiurazzi ${ }^{5,6^{*}}$ (D) and \\ Sylvia A. Frazier-Bowers ${ }^{7}$
}

\begin{abstract}
Background: Primary failure of eruption (PFE) is a hereditary condition, and linkage with variants in the PTH1R gene has been demonstrated in many cases. The clinical severity and expression of PFE is variable, and the genotype-phenotype correlation remains elusive. Further, the similarity between some eruption disorders that are not associated with PTH1R alterations is striking. To better understand the genotype-phenotype correlation, we examined the relationship between the eruption phenotype and PTH1R genotype in 44 patients with suspected PFE and 27 unaffected relatives. Sanger sequencing was employed to analyze carefully selected PFE patients. Potential pathogenicity of variants was evaluated against multiple genetic databases for function prediction and frequency information.
\end{abstract}

Results: Mutational analysis of the PTH1R coding sequence revealed 14 different variants in 38 individuals (30 patients and 8 first-degree relatives), 9 exonic and 5 intronic. Their pathogenicity has been reported and compared with the number and severity of clinical signs. In $72.7 \%$ of patients with pathogenic variants, five clinical and radiographic criteria have been found: involvement of posterior teeth, involvement of the distal teeth to the most mesial affected, supracrestal presentation, altered vertical growth of the alveolar process and posterior open-bite. In cases with mixed dentition (3), the deciduous molars of the affected quadrant were infraoccluded.

Discussion: The probability of an affected patient having a PTH1R variant is greater when five specific clinical characteristics are present. The likelihood of an eruption defect in the absence of specific clinical characteristics is rarely associated with a PTH1R mutation.

Conclusions: We report here that systematic clinical and radiographic observation using a diagnostic rubric is highly valuable in confirming PFE and offers a reliable alternative for accurate diagnosis.

Keyword: Primary failure of eruption, Orthodontics, PTH1R gene, Dental eruption, PFE diagnosis

\section{Background}

Tooth eruption is a complex process that represents an integral part of the broader tooth developmental process [1]. It is known that the tooth follicle interacts with both osteoblasts and osteoclasts during the tooth eruption

\footnotetext{
*Correspondence: pietro.chiurazzi@unicatt.it

${ }^{5}$ Dipartimento Universitario Scienze della Vita e Sanità Pubblica, Sezione di Medicina Genomica, Università Cattolica del Sacro Cuore, Largo

Francesco Vito 1, 00168 Rome, Italy

Full list of author information is available at the end of the article
}

process $[2,3]$. To date, however, neither the eruption mechanism nor the factors controlling eruption are completely understood $[4,5]$.

Eruption disorders are ideally classified based on their etiology; those secondary to obstruction (cysts, ankyloses, lateral tongue pressure, impaction, etc.), or those with genetic underpinnings (e.g., primary failure of eruption (PFE); cleidocranial dysplasia, Hunter's disease and osteopetrosis) should be considered [6]. 
Among the more common diagnostic distinctions, mechanical failure of eruption (MFE), ankylosis and primary failure of eruption (PFE) are examples of nonsyndromic eruption disorders, but with vastly different clinical implications. MFE is defined as eruption failure due to mechanical obstruction of the eruption pathway, which can be treated successfully with the removal of the obstruction. Ankylosis is defined as the fusion of cementum to alveolar bone, but the practical diagnosis of ankylosis relies on the clinical appearance of infraocclusion and the absence of a periodontal ligament in radiographs [6-8]. In case of an early diagnosis of a first molar's ankylosis, the therapy consists in the extraction of the ankylosed tooth and probably the second molar will drift mesially and will erupt [9].

Primary failure of eruption (OMIM \#125350) was first described by Proffit and Vig [10] as a condition in which non-ankylosed teeth fail to erupt. Involved teeth can erupt partially and then cease to erupt, becoming relatively submerged although not being ankylosed. Posterior teeth are also affected in PFE, resulting in a posterior open bite.

Diagnosis of PFE is extremely important to avoid the consequences of employing a continuous archwire [11]. Indeed, PFE-affected teeth have little or no response to orthodontic treatment with a tendency to encounter ankylosis or intrude adjacent teeth [9].

These broad categories of dental eruption failure can be distinguished based on the following diagnostic parameters: (1) patient dental history; (2) clinical and phenotypic characterization and; (3) genetic characterization. The well-documented patient dental history is critical and allows understanding the developmental trajectory through serial photographs and radiographs of the eruption sequence. For example, evaluation of the patient natural history is relevant for identifying or excluding MFE based on the identification of other pathology that may have caused a mechanical obstruction during a prescribed time period. Characteristic causes of MFE include dental crowding, the presence of cysts, and pathology resultant from trauma. In the absence of detailed clinical documentation over time, it is difficult to rule out mechanical obstruction as the cause for eruption failure. PFE can be distinguished from MFE and other eruption disorders by the use of a diagnostic rubric that provides clinical and phenotypic characterization [12, 13]. As with most causes of eruption failure, PFE can present in a unilateral or symmetrically bilateral pattern, with one or all posterior quadrants involved. Of the two described types of PFE, Type II can be easily mistaken for ankylosis as the second molar, by definition, shows more eruption that the first molar $[11,12,14]$. Another key characteristic of PFE in general is the abnormal or complete lack of response to orthodontic force, so that affected teeth cannot be moved into their proper position [10]. Despite the expanding base of knowledge describing the eruption disorders, there is a gap in the knowledge of the tooth eruption mechanism.

Although the presence of specific clinical signs allows suspicion of PFE, there is wide variability of phenotypic expression and the diagnosis can be established by sequencing the parathyroid hormone 1 receptor gene (PTH1R) [OMIM \#168468]. Ongoing genetic characterization of PFE is largely associated with autosomal dominant loss-of-function variants in the PTH1R gene, located on chromosome 3p21-p22.1, causing haploinsufficiency of the receptor [6, 10, 15-19]. Variants in PTH1R have also been associated with Jansen chondrodysplasia (OMIM \#156400), Blomstrand chondrodysplasia (OMIM \#215045), Eiken syndrome (OMIM \#600002) and Ollier enchondromatosis (OMIM \#166000). These conditions are characterized by abnormal skeletal development, and they are due either to recessive loss-of-function variants or a dominant gain-of-function variant in the PTH1R gene [20-23].

While it is helpful to analyze the PTH1R gene prior to treatment planning [15], merely establishing a family history through interviews and detailed clinical records on multiple family members is quite powerful. When analysis of the PTH1R gene is available, it represents an early diagnosis that is independent of family history $[6$, $10,25]$. In rare cases $P T H 1 R$ variants may be associated with incomplete penetrance [24, 26], further complicating the genotype-phenotype correlation. These and other deficiencies underscore the need to improve the gaps of knowledge and to explore the characteristics of an informative cohort.

Several studies in the literature have elevated the finding that specific clinical characteristics are correlated with functional alterations in the PTH1R gene [10, 1219]. In our report, we interrogate and validate that specific clinical characteristics $[12,13]$ correlate with the presence of PTH1R variants in a cohort of patients with suspected PFE and their relatives.

\section{Methods \\ Clinical analysis}

44 patients ( 26 males and 18 females), 23 in permanent dentition (age range 12-45 years) and 21 in mixed dentition (age range 5-12 years) with eruption disorders were enrolled in the Dental Clinic of the Policlinico "A. Gemelli" in Rome from November 2015 to May 2019. The study protocol was prepared in accordance with the Declaration of Helsinki. Ethical approval was obtained from the Ethics Committee of the Catholic University of Sacred Heart of Rome (study ID 565-11/2015). Patients 
with suspected PFE were evaluated by three qualified orthodontists including clinical examination, intraoral photographs and orthopanoramic radiography. Assessment of clinical signs was established with the consent of all three examiners, on a case-by-case basis (see Tables 3, 4, 5 for included characteristics). Patients were included in the study if at least one infraoccluded permanent molar was reported in permanent dentition, or one or more infraoccluded deciduous molar were observed in mixed dentition.

All enrolled patients were examined clinically and provided a saliva sample after consenting to study participation. DNA was extracted from saliva and subjected to PCR and sequencing as described below. Our analysis included 27 unaffected relatives of affected individuals.

\section{PTH1R sequencing}

DNA was collected using a cytobrush (Cooper Surgical, Trumbull, CT, USA) and extracted and purified with the 401 Genomic DNA Tissue Kit (MagCore ${ }^{\circledR}$, RBC Bioscience Corp.). Amplification of all coding exons of PTH1R [AB 3500 Genetic Analyzer (Life Technologies, Carlsbad, CA, USA)] was carried out following quantification with the Qubit Fluorometer (Life Technologies, Carlsbad, CA, USA) and verification of DNA integrity with agarose gel electrophoresis as previously described [25]. Primers employed for sequencing the coding sequence and exon-intron junctions of the PTH1R gene are reported in Table 1.

\section{Analysis of variants}

All variants have been positioned on the GRCh37/hg19 human genome assembly, and their frequency in the general population was retrieved from the Genome Aggregation Database (GnomAD) [27] and the Single Nucleotide Polymorphism Database (dbSNP) [28]. In order to evaluate the potential pathogenicity of PTH1R variants, we looked them up in the NCBI ClinVar database [29] and in the Leiden Open Variation database [30]. Finally, we cross-checked each variant using VarSome [31], a powerful annotation tool and search engine for human genomic variants [32].

\section{Statistical analysis}

Descriptive statistics were calculated. The frequency of PFE key clinical traits was analyzed using Graph Pad Prism program (https://www.graphpad.com/scientificsoftware/prism/). Fisher's exact test was employed to evaluate the significant association between variables, i.e., group with PTH1R (likely) pathogenic variants, group with $P T H 1 R$ benign variants and group without $P T H 1 R$ variants.
Table 1 Primers employed for sequencing PTH1R coding exons

\begin{tabular}{|c|c|}
\hline PTH1R exon & Primer sequence \\
\hline 3 FORWARD & 5'-AGCCTGACGCAGCTCTGCA-3' \\
\hline 3 REVERSE & $5^{\prime}-C C C A C A G T C C A G A C A T C C C A-3^{\prime}$ \\
\hline 4 FORWARD & 5'-AGAGCAGATTCCCCACATGC-3' \\
\hline 4 REVERSE & 5'-TTCACCTGGCTCTGTATCCT-3' \\
\hline 5 FORWARD & 5'-TCCTCACCCATCGTCTCAGAT-3' \\
\hline 5 REVERSE & $5^{\prime}$-AAGAGCCAAGAAGCATGAGC-3' \\
\hline 6 FORWARD & 5'-AGATGTATTCATCCTTCTGGG-3' \\
\hline 7 REVERSE & 5'-TAAGGTTGCTGGAGGAGTCAAG-3' \\
\hline 8 FORWARD & 5'-AAATTCACTCCCACCCCACG-3' \\
\hline 8 REVERSE & 5'-TGGACAGGAAGCTGGGTTGT-3' \\
\hline 9 FORWARD & 5'-ACAACCCAGCTTCCTGTCCA-3' \\
\hline 9 REVERSE & 5'-GTTGCGAGGGACCCTATAAG-3' \\
\hline 10 FORWARD & 5'-AAACGAAGCCTGCCCCTTC-3' \\
\hline 10 REVERSE & 5'-GCCTGGAATAGGGTCAGGAT-3' \\
\hline 11 FORWARD & $5^{\prime}$-GGAATGACCTTGTGGACAGC-3' \\
\hline 11 REVERSE & 5'-TAGCTGTTGAGGACACAGGG-3' \\
\hline 12 FORWARD & 5'-AGGGTCACAGGAGGCTACTT-3' \\
\hline 12 REVERSE & 5'-TGTCACTGCATCTCTGGGTG-3' \\
\hline 13 FORWARD & $5^{\prime}-C C A G C C C A G A A A G G A A A A C C-3^{\prime}$ \\
\hline 13 REVERSE & 5'-TAGTGCAGGGCCTGGTACAA-3' \\
\hline 14 FORWARD & 5'-AGGTGAACTGGGTTGTCCTC-3' \\
\hline 15 REVERSE & 5'-GAATGTCCTCAGGGGTGTTC-3' \\
\hline 16 FORWARD & 5'-CACTTGGCCTTGGAGTTTCC-3' \\
\hline 16 REVERSE & 5'-CCACCCATCTTTTGGTCC-3' \\
\hline
\end{tabular}

\section{Results}

\section{Genetic results}

Molecular analysis of the PTH1R coding sequence revealed 14 different variants in 38 affected individuals (27 patients and 11 first-degree relatives). Table 2 summarizes all variants found in our cohort (9 exonic and 5 intronic) along with their relative frequency in heterozygous and homozygous subjects from the general population as reported in the Genome Aggregation Database. The 9 exonic PTH1R variants had different effects on the protein structure: 1 nonsense variant, 1 frameshift variant and 4 missense variants, all not reported in the control population; 3 synonymous variants, two of which are reported in GnomAD. The 5 intronic variants have no obvious effect on splicing and all but one are very frequent in normal control individuals.

The nonsense variant, c.505G $>\mathrm{T}-\mathrm{p}$. (Glu169Ter), not reported in any genomic databases, was identified in patient 5 and his mother (ID.16), who has no clinical signs of PFE. This variant introduces a premature stop codon early in the coding sequence and is classified as Class 5 (pathogenic), possibly resulting in nonsensemediated decay and complete absence of the protein. 
Table 2 PTH1R variants found in our cohort with their CDNA and genomic location, predicted effect and classification, CADD scores for exonic variants, and frequency in gnomAD

\begin{tabular}{|c|c|c|c|c|c|c|c|c|c|}
\hline Patients ID & $\begin{array}{l}\text { Variant } \\
\text { NM_001184744.1 }\end{array}$ & $\begin{array}{c}\text { Genomic location } \\
\text { GRCh37/hg19 }\end{array}$ & Predicted effect & $\begin{array}{c}\text { Classification } \\
\text { (varsome.com) }\end{array}$ & CADD score & $\begin{array}{c}\text { Alleles with } \\
\text { variant }\end{array}$ & Total alleles & $\begin{array}{c}\text { Homozygotes } \\
\text { for variant }\end{array}$ & $\begin{array}{c}\text { Variant } \\
\text { frequency }\end{array}$ \\
\hline $5-16$ & $\begin{array}{l}\text { c.505G }>\mathrm{T} \\
\text { exon } 7\end{array}$ & chr3-46939644 & $\begin{array}{c}\text { p.Glu169Ter } \\
\text { nonsense }\end{array}$ & $\begin{array}{c}\text { Class } 5 \text { - } \\
\text { Pathogenic }\end{array}$ & 39.0 & 0 & 0 & 0 & 0 \\
\hline 17 & $\begin{array}{l}\text { c.1595delC } \\
\text { exon } 16\end{array}$ & chr3-46944957 & $\begin{array}{c}\text { p.Pro532Leufs* } \\
\text { frameshift }\end{array}$ & $\begin{array}{c}\text { Class } 5 \text { - } \\
\text { Pathogenic } \\
\end{array}$ & 24.1 & 0 & 0 & 0 & 0 \\
\hline 19 & $\begin{array}{l}\text { c.64G>T } \\
\text { exon } 3\end{array}$ & chr3-46925113 & $\begin{array}{l}\text { p.Ala22Val } \\
\text { missense }\end{array}$ & $\begin{array}{l}\text { Class } 3 \text { - uncertain } \\
\text { significance (VUS) }\end{array}$ & 24.7 & 0 & 0 & 0 & 0 \\
\hline $11-12$ & $\begin{array}{l}\text { c.337G }>A \\
\text { exon } 6\end{array}$ & chr3-46939368 & $\begin{array}{l}\text { p.Asp113Asn } \\
\text { missense }\end{array}$ & $\begin{array}{c}\text { Class } 4 \text { - } \\
\text { Likely pathogenic }\end{array}$ & 26.4 & 0 & 0 & 0 & 0 \\
\hline $3-4-29$ & $\begin{array}{l}\text { c. } 1765 T>C \\
\text { exon } 16\end{array}$ & chr3-46945129 & $\begin{array}{l}\text { p.Trp589Arg } \\
\text { missense }\end{array}$ & $\begin{array}{l}\text { Class } 3 \text { - uncertain } \\
\text { significance (VUS) }\end{array}$ & 23.0 & 0 & 0 & 0 & 0 \\
\hline 6 & $\begin{array}{l}\text { c. } 216 \mathrm{G}>\mathrm{A} \\
\text { exon } 5\end{array}$ & chr3-46937262 & $\begin{array}{c}\text { p.Ala72= } \\
\text { synonymous }\end{array}$ & $\begin{array}{c}\text { Class } 2 \text { - } \\
\text { Likely benign }\end{array}$ & 0.44 & 510 & 282860 & 3 & $1.8 \mathrm{e}-3$ \\
\hline $\begin{array}{l}1-2-3-4-13-22- \\
29\end{array}$ & $\begin{array}{l}\text { c. } 951 \mathrm{G}>\mathrm{A} \\
\text { exon } 10\end{array}$ & chr3-46940909 & $\begin{array}{l}\text { p.Glu317= } \\
\text { synonymous }\end{array}$ & $\begin{array}{c}\text { Class } 2 \text { - } \\
\text { Likely benign }\end{array}$ & 22.5 & 0 & 0 & 0 & 0 \\
\hline $\begin{array}{l}1-6-7-10-12-14- \\
24-19-37 \\
\text { (heterozygotes) } \\
8-9-30 \\
\text { (homozygotes) }\end{array}$ & $\begin{array}{l}\text { c. } 1389 T>C \\
\text { exon } 15\end{array}$ & chr3-46944274 & $\begin{array}{l}\text { p.Asn463= } \\
\text { synonymous }\end{array}$ & Class 1 - benign & 18.5 & 172208 & 280652 & 53758 & $6.14 \mathrm{e}-1$ \\
\hline $23-37-43-49$ & $\begin{array}{l}\text { c. } 178+28 G>A \\
\text { intron } 4\end{array}$ & chr3-46935527 & Intronic & $\begin{array}{l}\text { Class } 3 \text { - uncertain } \\
\text { significance (VUS) }\end{array}$ & - & 6 & 247816 & 0 & $2.42 e-5$ \\
\hline $17-24$ & $\begin{array}{l}\text { c. } 313+32 A>G \\
\text { intron } 5\end{array}$ & chr3-46937391 & Intronic & Class 1 - benign & - & 5885 & 270764 & 82 & $2.17 e-2$ \\
\hline $17-18-72$ & $\begin{array}{l}\text { c. } 543+14 \mathrm{~T}>\mathrm{C} \\
\text { intron } 7\end{array}$ & chr3-46939696 & Intronic & Class 1 - benign & - & 6253 & 282780 & 103 & $2.21 \mathrm{e}-2$ \\
\hline 24 & $\begin{array}{l}\text { c. } 638+46 \text { dupG } \\
\text { intron } 8\end{array}$ & chr3-46940008 & Intronic & Class 1 - benign & - & 6103 & 269332 & 97 & $2.27 \mathrm{e}-2$ \\
\hline $\begin{array}{l}5-6-8-9-17-18- \\
23-24-47\end{array}$ & $\begin{array}{l}\text { c. } 1116+58 \mathrm{~T}>\mathrm{C} \\
\text { intron } 12\end{array}$ & chr3-46943028 & Intronic & Class 1 - benign & - & 15478 & 31200 & 4157 & $4.96 e-1$ \\
\hline \multicolumn{10}{|c|}{ : Intronic variants (most likely benign) } \\
\hline \multicolumn{10}{|c|}{ : Synonymous variants (benign/likely benign/uncertain significance) } \\
\hline \multicolumn{10}{|c|}{ : Missense variants (uncertain significance/likely pathogenic) } \\
\hline & \multicolumn{9}{|c|}{ : Nonsense and frameshift variants (likely pathogenic/pathogenic) } \\
\hline
\end{tabular}

The frameshift variant, c.1595delC-p.(Pro532Leufs*), due to the deletion of one cytosine in a short homopolymeric tract (1593-1595), results in the production of a longer protein with 21 extra amino acids at the C-terminus and is classified as Class 4 (likely pathogenic).

Two of the 4 missense variants were previously reported [24], namely c.64G $>\mathrm{T}-$ p.(Ala22Val) and c. $1765 \mathrm{~T}>\mathrm{C}-\mathrm{p}$.(Trp589Arg), and are classified as variants of unknown significance (VUS/Class 3). The c.64G>T causes the replacement of alanine in position 22 with a valine in the extracellular domain of the protein and was found in a very young patient (ID.19) with mixed dentition. The c.1765T $>\mathrm{C}$ was found in two sibs and their mother (ID. 3-4-29). This variant replaces tryptophan 589 with arginine at the end of the protein in the intracellular domain of the receptor.

Two other missense variants, c.337G $>A-p$. (Asp113Asn) and c.354G>T-p.(Trp118Cys), have never been previously reported and are both classified as likely pathogenic (Class 4). The c.337G>A results in aspartic acid to asparagine change in position 113 in the extracellular domain of the protein, and was found in the proband (ID. 11) and in his mother (ID. 12). The c.354G $>$ T, resulting in the substitution of tryptophan 118 with cysteine, was detected in two brothers (ID. 14-15), whose parents were unavailable for clinical examination and genetic testing.

Only one of the synonymous variants found in our patients, c.951G $>A-$ p. (Glu317=), was absent in the control population (Class 3 , uncertain significance); interestingly this variant was also found in the two sibs and mother with the c.1765T $>C$ variant described above. Furthermore, the c.951G $>$ A was also found in a bilateral Type I PFE (ID. 2), in a unilateral Type 2 PFE (ID. 1), in a patient with failed eruption of upper and lower second molars (ID. 13), while the last patient (ID. 22) had a severe left open bite involving premolars and an included upper left canine.

Although the second synonymous variant, c.216G >Ap.(Ala72=), is reported in GnomAD with a frequency of $1.8 \times 10^{-3}$, suggesting its probable benignity, we found it in one patient (ID. 6) with bilateral Type I PFE.

The last synonymous variant, c.1389T $>\mathrm{C}-\mathrm{p}$. $($ Asn $463=)$, has an extremely high frequency and is reliably classified as benign.

All intronic variants found in our cohort do not change the splicing donor (GT) nor the acceptor (AG) sites and are unlikely to alter mRNA splicing. Four out of 5 


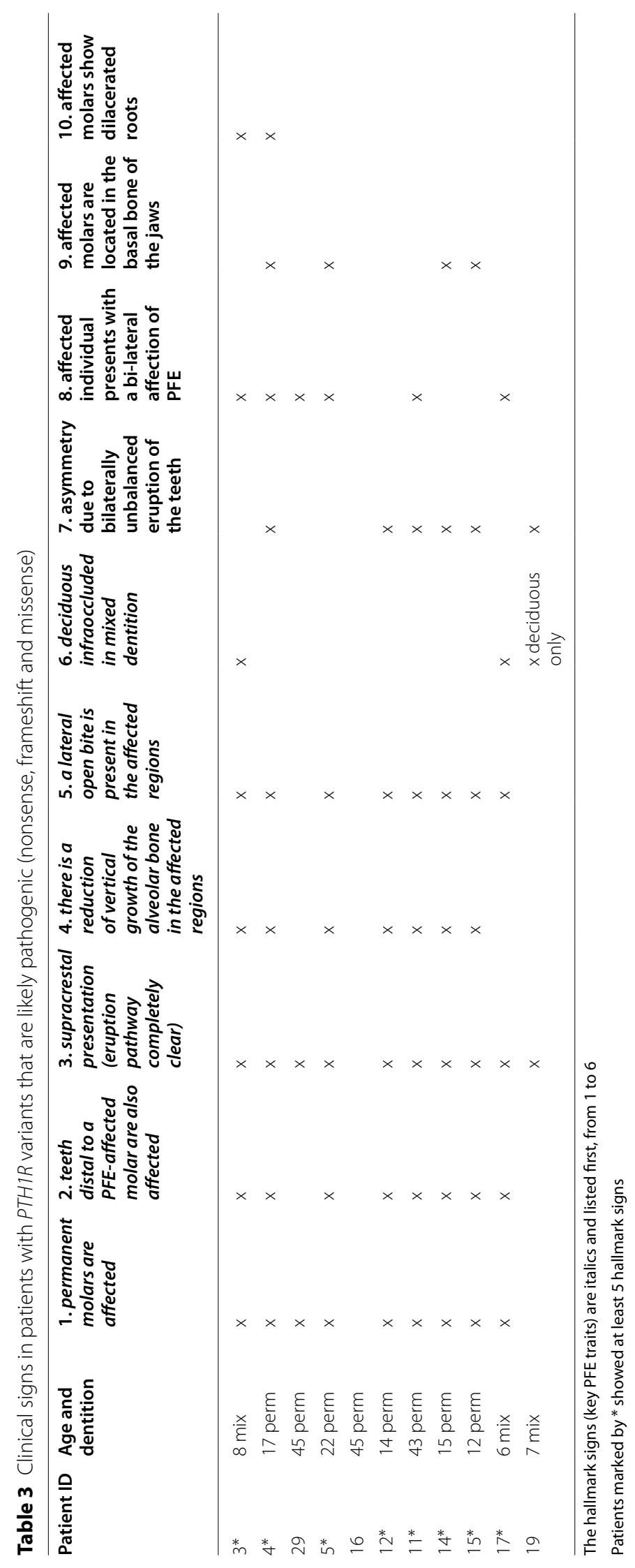




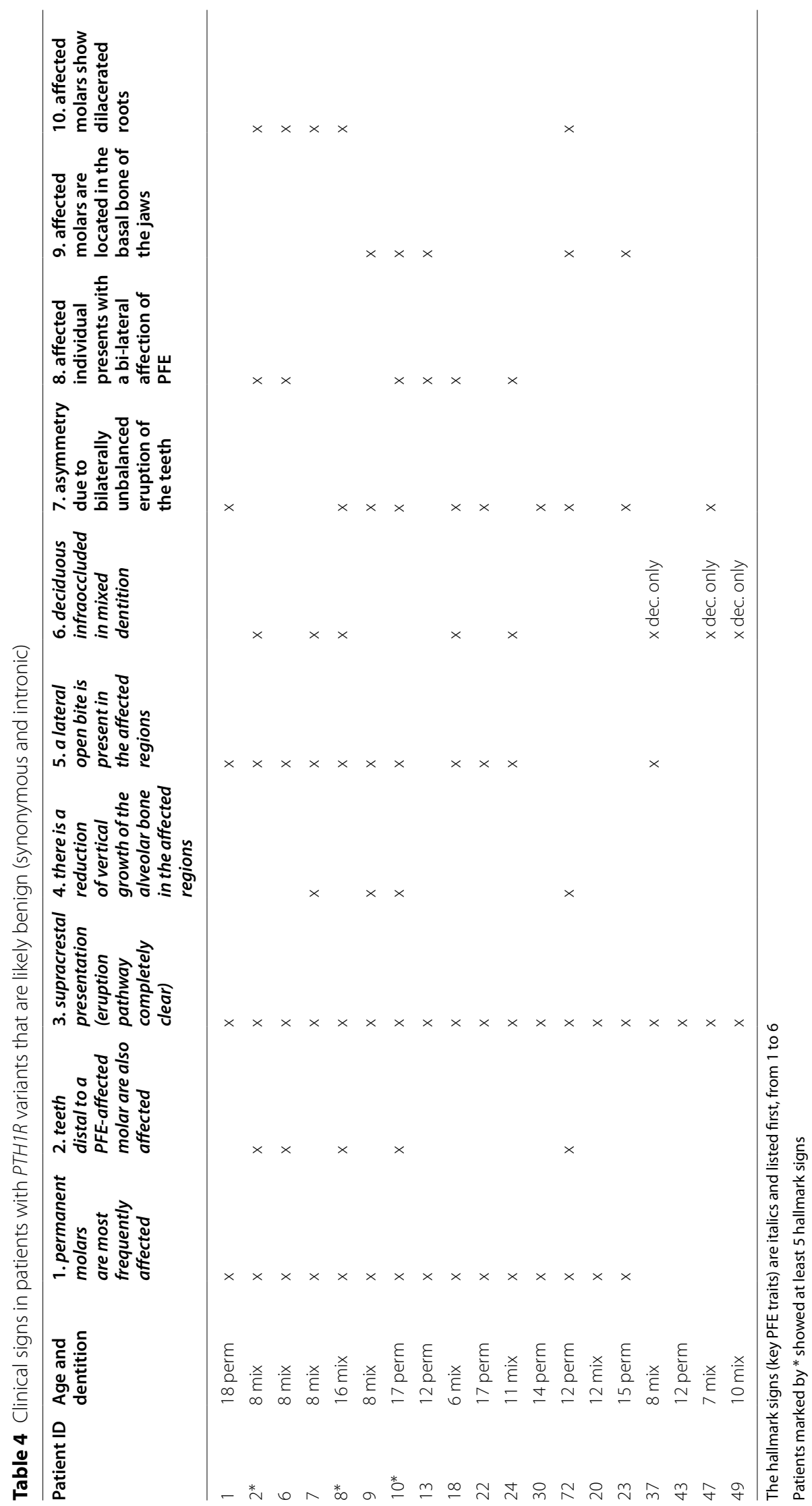




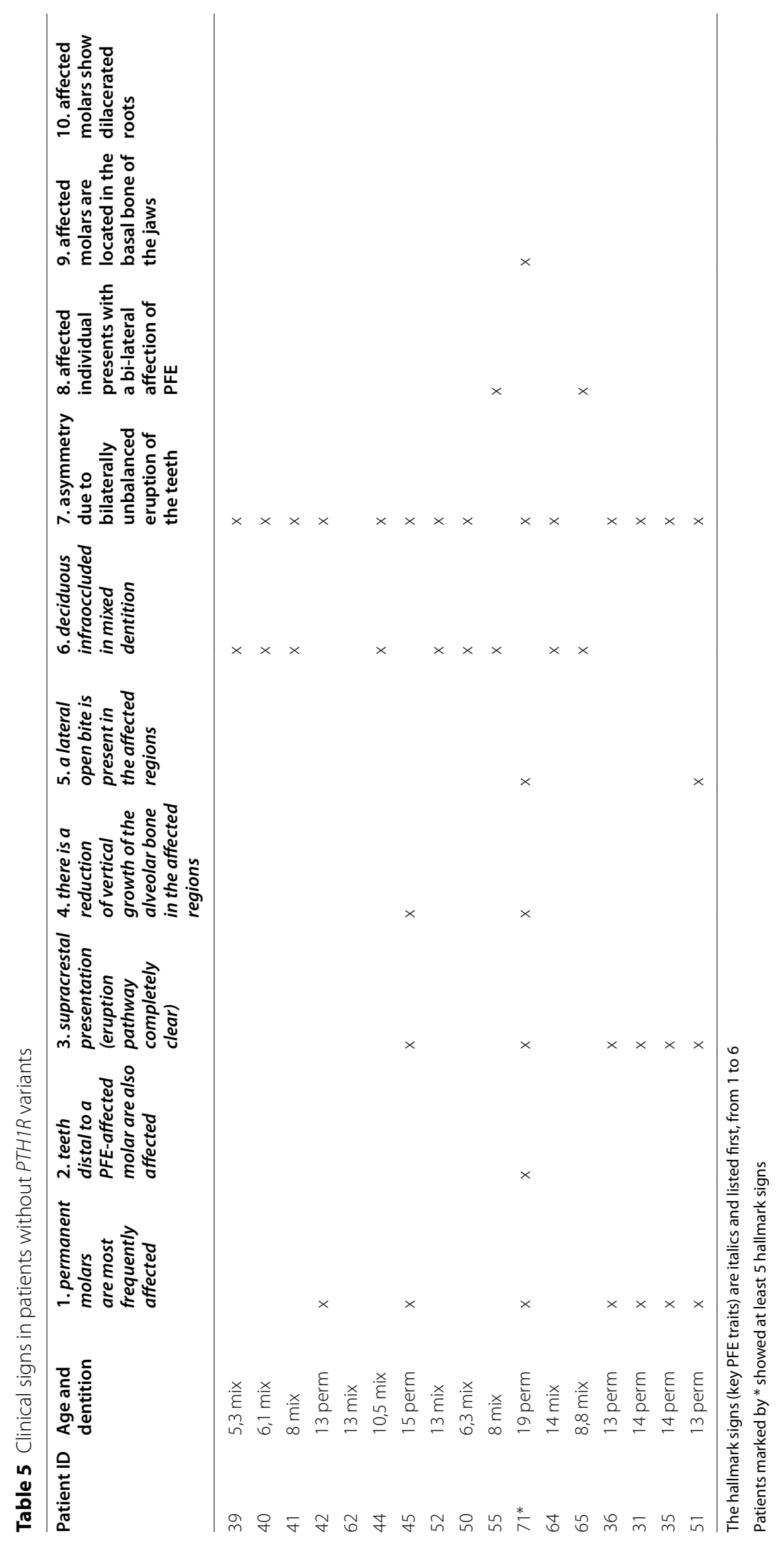



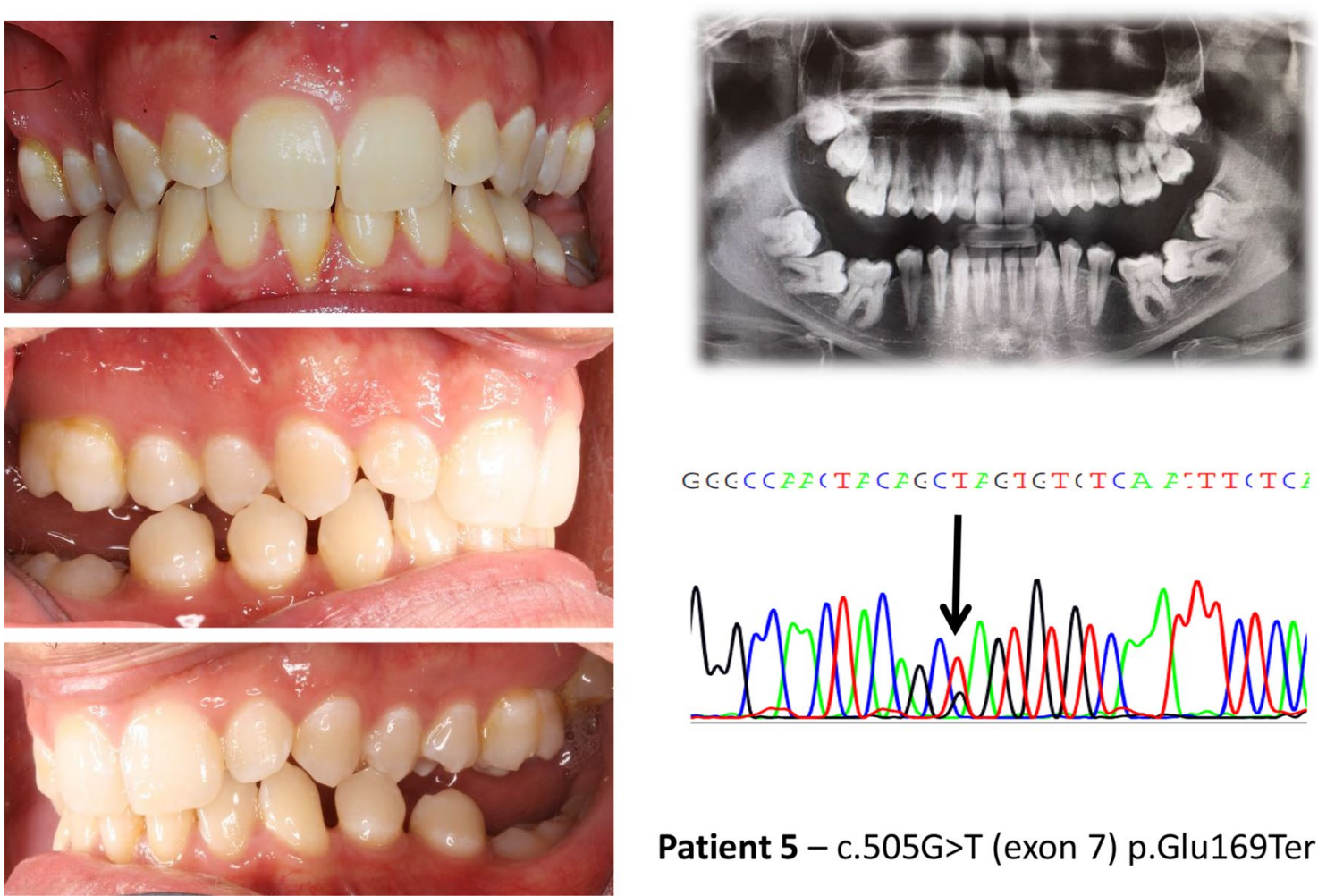

GCCCCFECTECFCCIICICTCTCA F TTCTCI

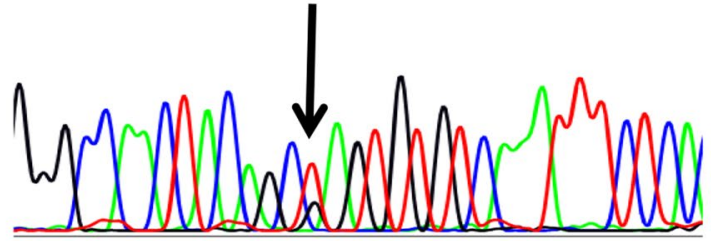

Patient 5 - c.505G>T (exon 7) p.Glu169Ter

Fig. 1 Clinical features and genetic findings of patient 5 with PFE and a PTHIR nonsense variant
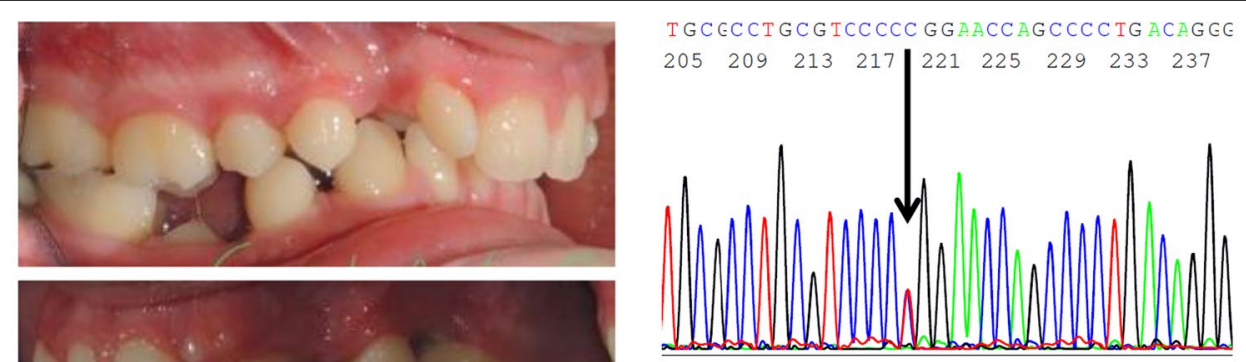

c. $1116+58 \mathrm{~T}>\mathrm{C}$ (intron 12)
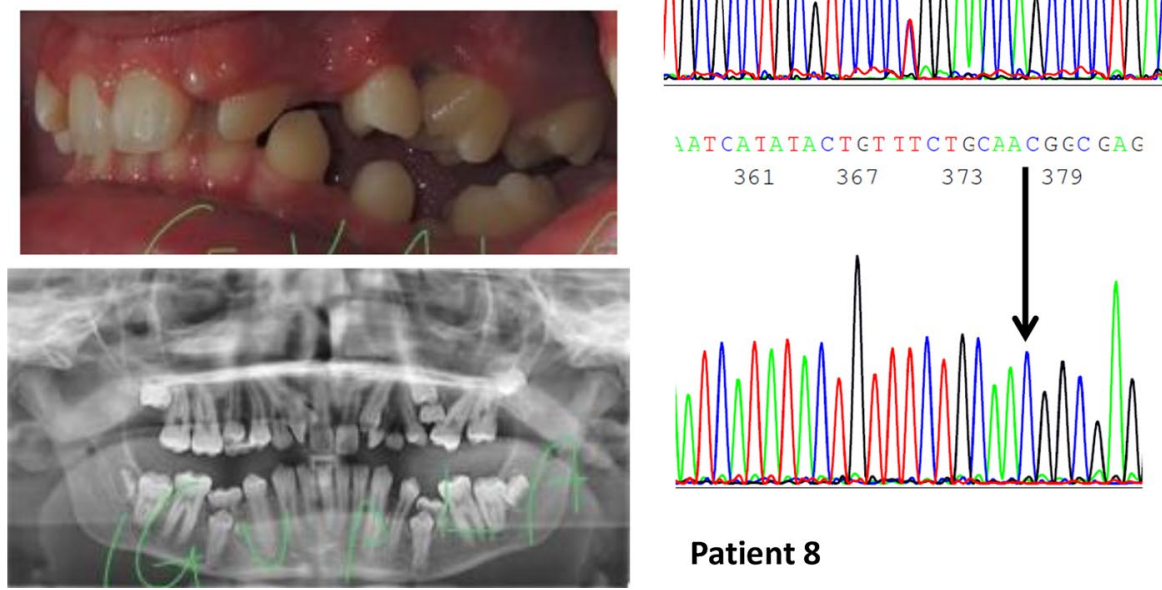

c.1389T $>$ C (exon 15) p.Asn463= homozygote

\section{Patient 8}

Fig. 2 Clinical features and genetic findings of patient 8 with PFE and both a synonymous and an intronic PTH1R variant 


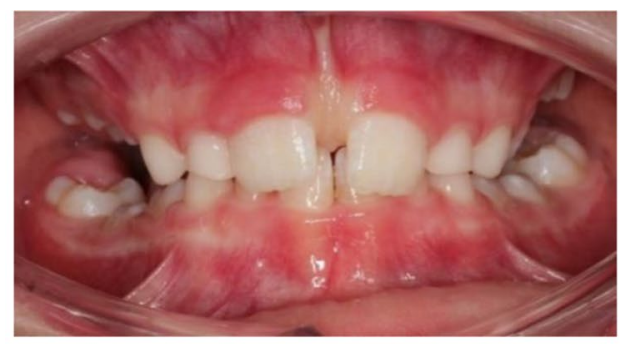

\section{CT TCTTCTCAGAAAAGAAGIACCTG \\ $\begin{array}{lllll}05 & 211 & 217 & 223 & 229\end{array}$}
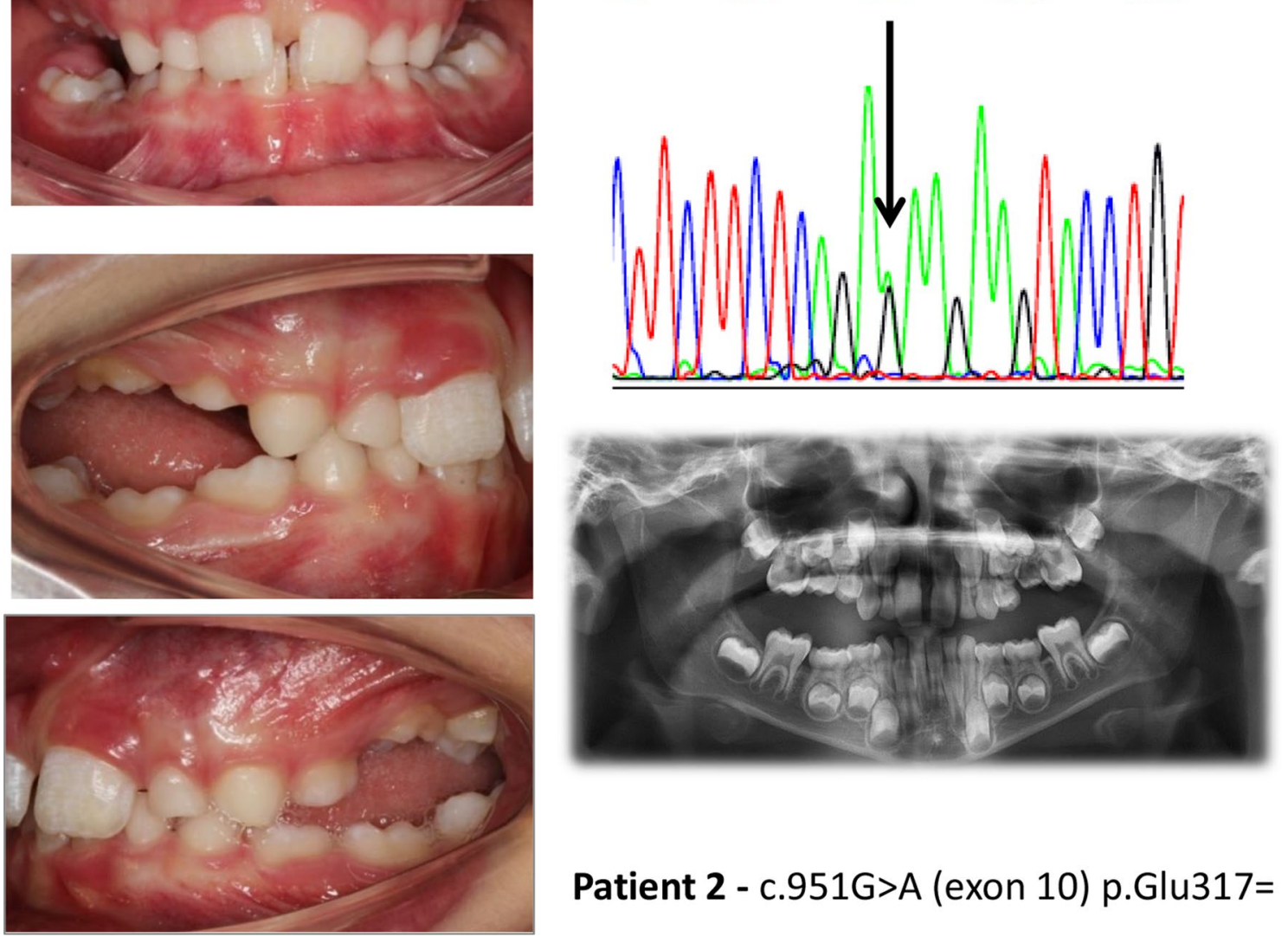

Patient 2 - c.951G>A (exon 10) p.Glu317=

Fig. 3 Clinical features and genetic findings of patient 2 with PFE and a synonymous PTH1R variant

of the identified intronic variants are indeed quite frequent in the control population and can be considered benign (Class 1) common polymorphisms. Only the c. $178+28 \mathrm{G}>\mathrm{A}$, found in intron 4 , has a very low frequency $\left(2.42 \times 10^{-5}\right)$ and is considered a VUS (Class 3$)$, as indicated in Table 2.

\section{Clinical and radiographic findings}

Genetic analysis allowed us to divide our patients into three groups: 11 patients ( 6 male and 5 female), in whom heterozygous nonsense, frameshift and missense PTH1R variants were found; 19 patients (12 male and 7 female) with synonymous and intronic variants, and 17 patients (8 male and 9 female) without PTH1R variants. In Tables 3, 4 and 5, we categorized patients on the basis of their clinical and radiographic findings in order to highlight the genotype-phenotype correlation.

In patients with PTH1R variants (nonsense, frameshift and missense) that alter the protein structure (Table 3), the open bite is more severe and Type I PFE is frequent. In this group of patients, 8 out of $11(72,7 \%)$ showed all the typical signs of PFE reported by Pilz et al. [13] in $100 \%$ of their patients with pathogenic PTH1R variants (Fig. 1). One patient (ID. 19), carrying the missense variant c.64G $>\mathrm{T}$, had a very mild phenotype at 8 years of age but was lost to follow-up for further characterization. Our clinical characterization also revealed a disconnect in the eruption phenotype among 3 parent-child pairs carrying the same PTH1R change. While three mothers of children with clinical signs of PFE also carried the variant, their corresponding phenotype varied. One mother had a normal phenotype, the second had a unilateral open bite (corrected by prosthetic crowns) and the third showed the inclusion of an upper canine and a lower second premolar (not typical of PFE). Notably the relative infraocclusion of the molar teeth was insignificant.

In patients with synonymous and intronic PTH1R variants (Table 4), whose functional effect is uncertain, only three out of 19 had typical PFE. In these three patients, we noticed the presence of all the most typical signs of PFE. One was very atypical, with premolar inclusions, but the other two looked as typical PFE (Figs. 2, 3, 4). In the 

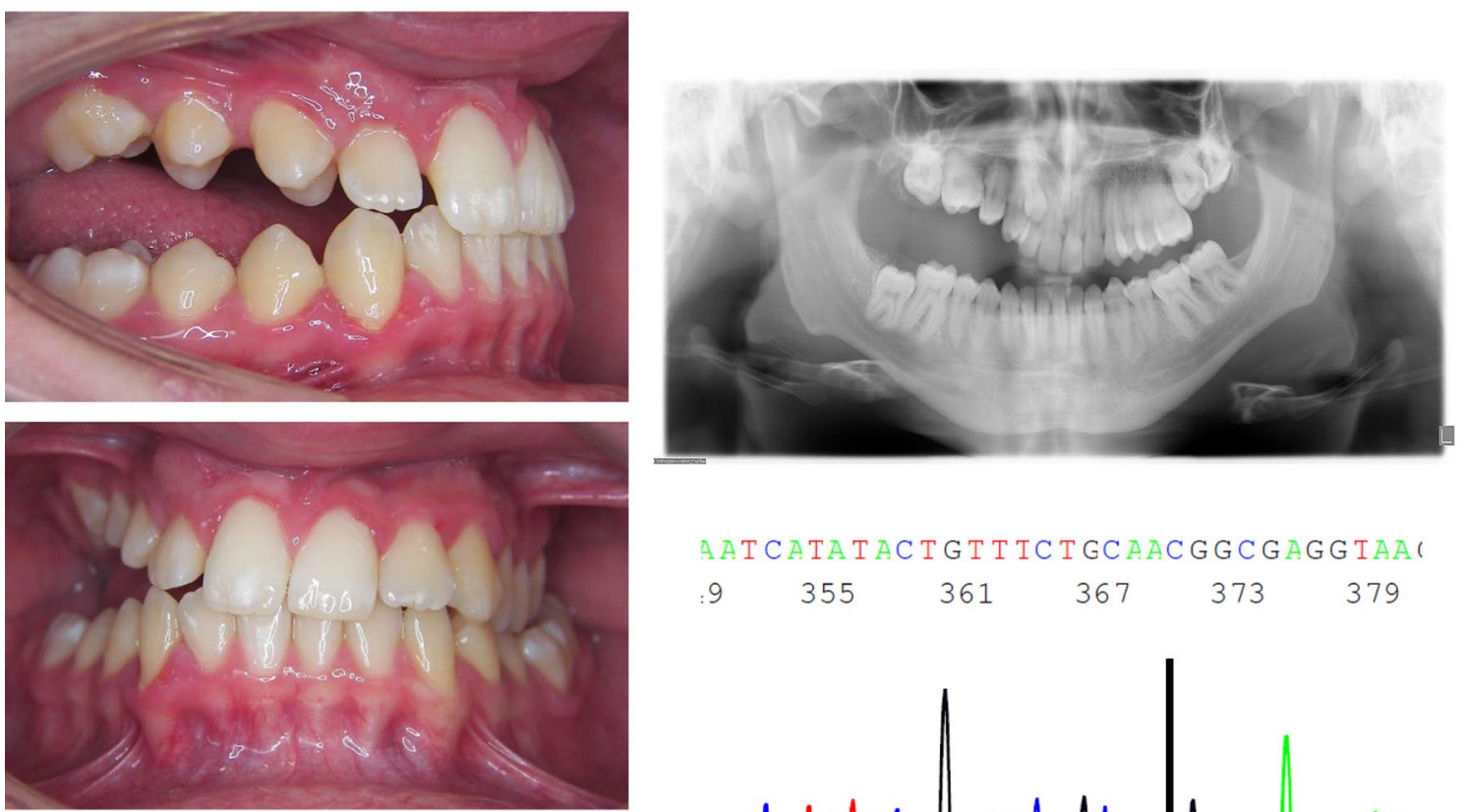

A A T ATATACTGTTICTGCAACGGCGAGGIAA

$\begin{array}{llllll}9 & 355 & 361 & 367 & 373 & 379\end{array}$
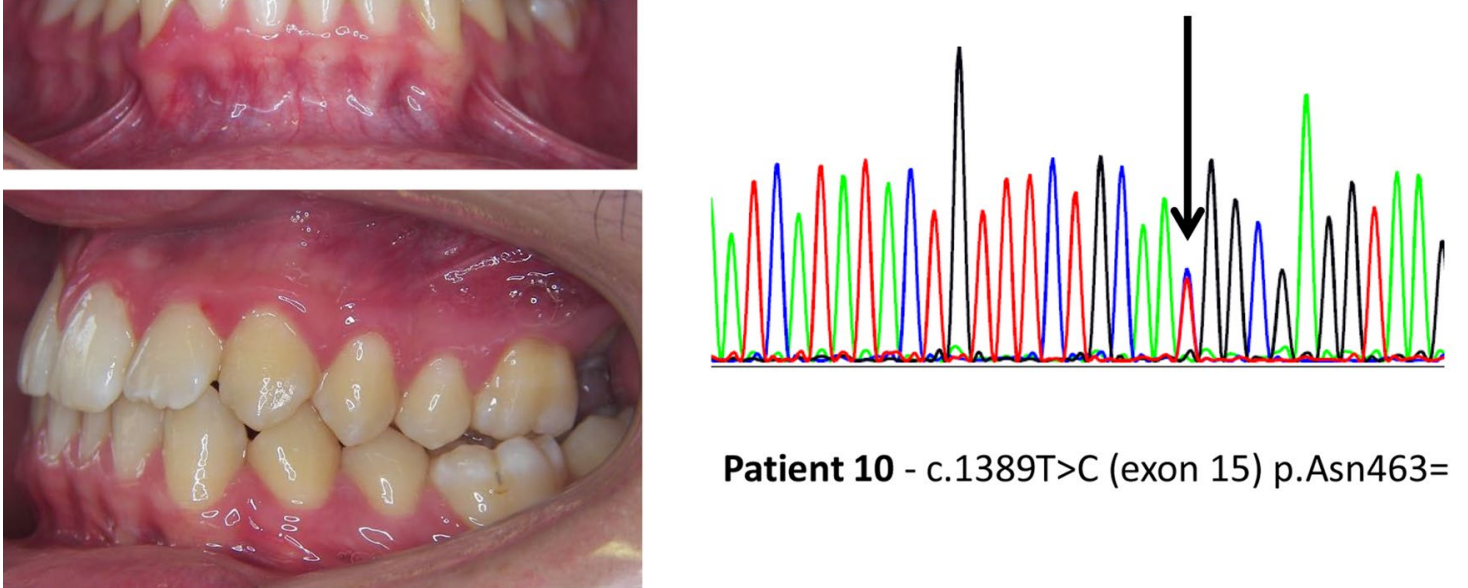

Patient 10 - c.1389T $>C$ (exon 15) p.Asn463=

Fig. 4 Clinical features and genetic findings of patient 10 with PFE and an intronic PTH1R variant

third group (Table 5), without PTH1R variants, only one out of 17 had all typical PFE signs. The diagnosis based on clinical results eventually allowed us to exclude the diagnosis of PFE, and the results of the genetic test confirmed it. The only patient of this group who had a severe clinical phenotype, showed the first upper right molar infraoccluded and the second and third molars included, and a history of orthodontic therapy failure (Fig. 5).

\section{Statistical results}

As indicated in Fig. 6, from a total of 11 subjects with pathogenic PTH1R variants, 5 or more clinical hallmarks (key traits) of PFE were present in 8 of them (72.7\%). Among the group of 19 subjects with benign PTH1R variants, 5 or more hallmarks were observed in $3(15.8 \%)$, while in the group without variants (17 subjects) only 1 showed all 5 key traits (5.9\%). Fisher's exact test showed a significant association between the presence of the key clinical traits in the group with pathogenic variants compared to the group with benign variants $(p=0.0045)$, and to the group without variants $(p=0.0004)$, and to the groups with benign variants and without variants pooled together $(p=0.0002)$. The comparison between the group with benign variants versus the group without PTH1R variants showed no significant difference $(p=0.6052)$, as expected.

\section{Discussion}

A total of 44 patients and 3 mothers were sequenced in this study, of which 30 were genetically identified as carriers of variants of the PTH1R gene, while in the other 17 no variants were found. The variant carrier group included 18 males and 12 females, although PFE cases are not known to have a gender difference [13]. The noncarrier group included 8 males and 9 females, again suggesting no gender difference. As detailed in Table 3, 11 of the 30 patients carrying pathogenic or likely pathogenic PTHR1 variants had mostly bilateral Type I PFE, while 

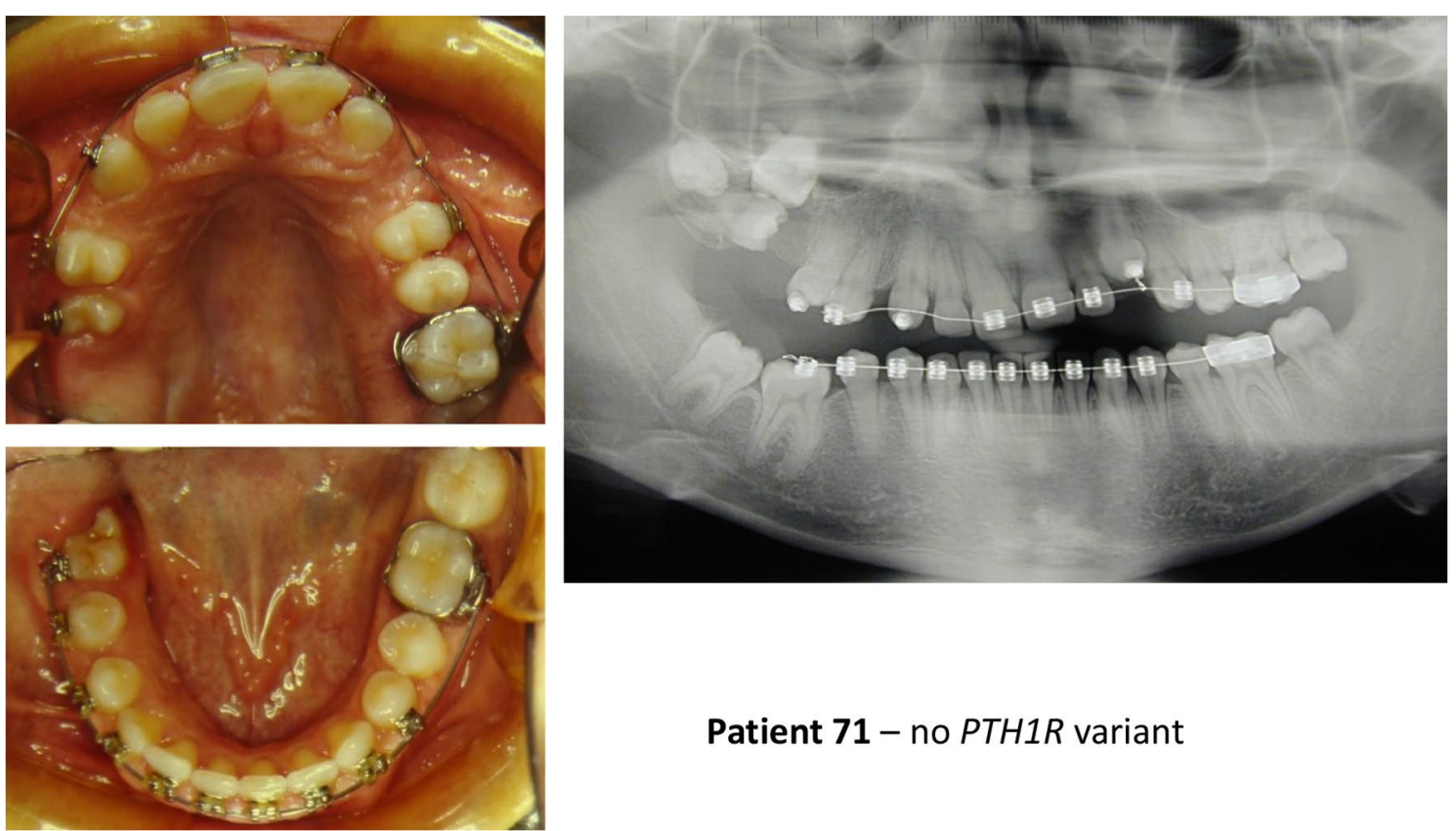

Fig. 5 Clinical features and genetic findings of patient 71 with PFE but without PTH1R variants

\section{Patient 71 - no PTH1R variant}

\begin{tabular}{|c|c|c|c|c|}
\hline & (likely) Pathogenic & (likely) Benign & no PTH1R variants & \\
\hline $5+$ key PFE traits present & 8 & 3 & 1 & 12 \\
\hline \multirow[t]{2}{*}{$<5$ key traits present } & 3 & 16 & 16 & 35 \\
\hline & 11 & 19 & 17 & 47 \\
\hline
\end{tabular}

prevalence of 5 or more key PFE traits in patients with/out $P T H 1 R$ gene variants

20

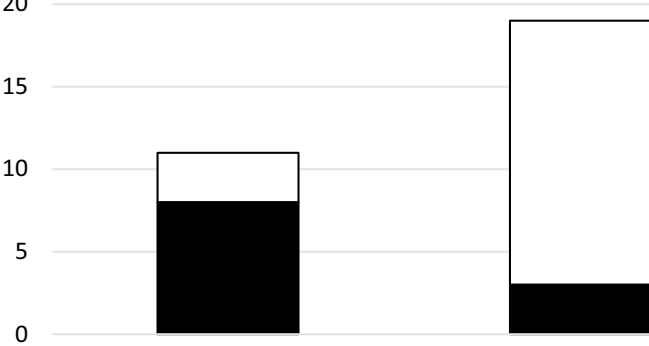

(likely) Pathogenic

(likely) Benign

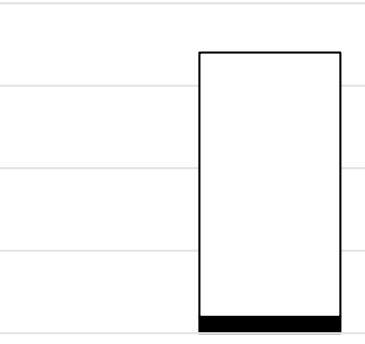

no PTH1R variants

- 5+ key PFE traits present

$\square<5$ key traits present

\begin{tabular}{|l|c|}
\multicolumn{1}{c}{ Fisher exact test } & two-tailed P value \\
\hline Path vs. Benign & $0.0045^{*}$ \\
\hline Path vs. no variant & $0.0004^{* *}$ \\
\hline Benign vs. no variant & 0,6052 \\
\hline Path vs. Benign+no variant & $0.0002^{* *}$ \\
\hline
\end{tabular}

Fig. 6 Statistical analysis and significant association of 5 or more PFE key traits (hallmark signs) with (likely) pathogenic PTH1R variants 
asymmetrical involvement was more frequent in the 19 patients with benign or likely benign PTH1R variants. Molar root dilacerations were very rare, and the other clinical signs did not discriminate the pathogenicity of the PTH1R variants. The remaining 17 patients without PTH1R variants, with few notable exceptions, showed a less defined phenotype, sometimes limited to the involvement of a single dental element, when compared to PTH1R-positive patients.

Five clinical and radiographic criteria described by Pilz et al. [13] have been found in almost all carriers of PTH1R variants: involvement of posterior teeth, involvement of the distal teeth to the most mesial affected, supracrestal presentation (eruption pathway completely clear), altered vertical growth of the alveolar process and posterior open-bite. Statistical analysis (Fig. 6) showed a significant difference in the frequency of PFE key clinical traits between patients with (likely) pathogenic PTH1R variants and those with either benign or no variants. We therefore reject the null hypothesis of no association in favor of the alternative that a (non-zero) association exists in the population. In patients with pathogenetic variants of PTH1R and mixed dentition, one or more deciduous teeth were infraoccluded. Our data on deciduous dentition involvement are in agreement with Pilz et al. [13]. From the cohort reported here, we learned that systematic clinical and radiographic observations using a diagnostic rubric is highly valuable to validate the diagnosis of PFE. While genetic testing offers additional confirmation, toward confirming PFE, the rubric provided sufficient information for a correct diagnostic orientation, and for selecting patients eligible for genetic testing. However, a PTH1R variant was found only in $64 \%$ of PFE patients (30 of 47), and only $23 \%$ of these (11 out of 47) were carriers of pathogenic or likely pathogenic variants. The functional analysis of the effects of all PTH1R variants at the mRNA and protein level would be very useful, but it is challenging and has rarely been done [33-35]. We suspect that the phenotypic manifestation of PFE observed in the remaining patients might be due to variants in other genes, encoding other proteins involved in the complex process of tooth morphogenesis and eruption and not implicated in PFE pathogenesis yet [3-5]. The study of tooth morphogenesis, characterized by reciprocal epithelialmesenchymal interactions, has benefited from the use of animal models, primarily mice [36, 37]. For example, Philbrick et al. [36] first showed that PTH1R is highly expressed in the alveolar bone surrounding the dental follicle, while its ligand PTHrP is produced by cells of the enamel epithelium and the stellate reticulum and is necessary for the reabsorption of the alveolar bone overlying the erupting tooth. Therefore, patients with typical PFE characteristics, who do not carry pathogenic variants in the PTH1R gene, could be recruited for further genetic studies that seek, for example, alterations in the Parathyroid Hormone Like Hormone (PTHLH) gene coding for Parathyroid hormone-related protein (PTHrP) [38]. Indeed, Philbrick et al. [36] elegantly demonstrated that, in the mouse, expression of PTHrP by the enamel epithelium is necessary for the reabsorption of the bone roof overlying the dental elements, while its absence determines PFE. Furthermore, Klopocki et al. [39] highlighted the role of PTHrP in human dental eruption, observing that two out of five families affected by E1 type brachydactyly (OMIM \#113300) and carrying variants in the PTHLH gene, in addition to short stature and short fingers and metacarpals, also had multiple impacted teeth and/or PFE.

\section{Conclusion}

Our findings on a large PFE cohort suggest that the key clinical traits of PFE are significantly more present in patients with pathogenic variants of the PTH1R gene. Additionally, our descriptive statistic suggests that a subject who have signs of PFE may not have variants of the PTH1R gene. Considering our data and those described in the literature, we believe it is useful, whenever possible, to carry out the genetic test. When clinical signs are present and testing is not possible, the risks of undertaking orthodontic therapy in a patient with a high probability of PFE should be carefully considered.

From our results, the diagnostic rubric allows to select patients with high possibility of PFE with a pathogenic PTH1R variant. Furthermore, if $P T H 1 R$ variants are found, their pathogenic role should be questioned and at least an in silico analysis should be performed. If PTH1R variants are likely benign or are considered of uncertain significance, functional studies should be performed before linking any variant to the PFE phenotype. Finally, if PTH1R screening is negative and the alternative diagnoses of MFE or ankyloses have been excluded, we suggest to extend genetic testing at least to the PTHLH gene. In fact, considering the complex process of tooth formation and eruption and the multiple genes and corresponding proteins involved, the development of a dedicated gene panel would be highly desirable.

\section{Acknowledgements \\ Not applicable.}

\section{Authors' contributions}

CG contributed to conception of the work, patients' selection, drafted the work. IDA contributed to data acquisition. CC contributed to interpretation of data. AR contributed to laboratory procedures. PC contributed to interpretation of data. SAFB substantively revised the work. Each author approved the 
submitted version AND to have agreed both to be personally accountable for the author's own contributions and to ensure that questions related to the accuracy or integrity of any part of the work, even ones in which the author was not personally involved, are appropriately investigated, resolved, and the resolution documented in the literature. All authors read and approved the final manuscript.

\section{Funding}

This research did not receive any specific grant from funding agencies in the public, commercial, or not-for-profit sectors.

\section{Availability of data and materials}

The datasets used and/or analyzed during the current study are available from the corresponding author on reasonable request.

\section{Declarations}

Ethics approval and consent to participate

The study protocol was prepared in accordance with the Declaration of Helsinki. Ethical approval was obtained from the Ethics Committee of the Catholic University of Sacred Heart of Rome (study ID 565-11/2015). A written consent was collected for all the patients.

\section{Consent for publication}

Not applicable.

\section{Competing interests}

The authors declare that they have no competing interests.

\section{Author details}

${ }^{1}$ School of Dentistry, Università Cattolica del Sacro Cuore, L.go Agostino

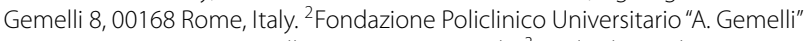
IRCCS, L.go Agostino Gemelli 8, 00168 Rome, Italy. ${ }^{3}$ Medical Oncology, S.G. Moscati, 74010 Statte, Taranto, Italy. ${ }^{4}$ Università Cattolica del Sacro Cuore, 00168 Rome, Italy. ${ }^{5}$ Dipartimento Universitario Scienze della Vita e Sanità Pubblica, Sezione di Medicina Genomica, Università Cattolica del Sacro Cuore, Largo Francesco Vito 1, 00168 Rome, Italy. ${ }^{6}$ UOC Genetica Medica, Fondazione Policlinico Universitario "A. Gemelli" IRCCS, Largo Agostino Gemelli 8, 00168 Rome, Italy. ${ }^{7}$ UNC Adams School of Dentistry, Chapel Hill, NC 27599, USA.

Received: 20 March 2021 Accepted: 23 October 2021 Published online: 13 December 2021

\section{References}

1. McCollum MA, Sharpe PT. Developmental genetics and early hominid craniodental evolution. BioEssays. 2001;23:481-93.

2. Wise GE, King GJ. Mechanism of tooth eruption and orthodontic tooth movement. J Dent Res. 2008;87:414-34.

3. Ono W, Sakagami N, Nishimori S, Ono N, Kronenberg HM. Parathyroid hormone receptor signalling in osterix-expressing mesenchymal progenitors is essential for tooth root formation. Nat Commun. 2016;7:11277.

4. Takahashi A, Nagata M, Gupta A, Matsushita Y, Yamaguchi T, Mizuhashi K, Maki K, Ruellas AC, Cevidanes LS, Kronenberg HM, Ono N, Ono W. Autocrine regulation of mesenchymal progenitor cell fates orchestrates tooth eruption. Proc Natl Acad Sci USA. 2019;116:575-80.

5. Cui C, Bi R, Liu W, Guan S, Li P, Song D, Xu R, Zheng L, Yuan Q, Zhou X, Fan $\mathrm{Y}$. Role of PTH1R signaling in $\mathrm{PrX}^{+}{ }^{+}$mesenchymal progenitors during eruption. J Dent Res. 2020;99:1296-305.

6. Frazier-Bowers SA, Puranik CP, Mahaney MC. The etiology of eruption disorders- further evidence of a genetic paradigm. Semin Orthod. 2010;16:180-5

7. Baccetti T. Tooth anomalies associated with failure of eruption of first and second permanent molars. Am J Orthod Dentofac Orthop. 2000;118:608-10.

8. Suri L, Gagari E, Vastardis H. Delayed tooth eruption: pathogenesis, diagnosis, and treatment. A literature review. Am J Orthod Dentofacial Orthop. 2004;126:432-45.
9. Frazier-Bowers SA, Long S, Tucker M. Primary failure of eruption-considerations for management by the orthodontist and oral surgeon. Semin Orthod. 2016;22:34-44.

10. Proffit WR, Vig KW. Primary failure of eruption: a possible cause of posterior open-bite. Am J Orthod. 1981;80:173-90.

11. Frazier-Bowers SA, Simmons D, Wright JT, Proffit WR, Akerman JL. Primary failure of eruption and PTH1R: the importance of a genetic diagnosis for orthodontic treatment planning. Am J Orthod Dentofacial Orthop. 2010;137(160):e1-7.

12. Rhoads SG, Hendricks HM, Frazier-Bowers S. Establishing the diagnostic criteria for eruption disorders based on genetic and clinical data. Am J Orthod Dentofacial Orthop. 2013;144:194-202.

13. Pilz P, Meyer-Marcotty P, Eigenthaler M, Roth H, Weber BHF, Stellzig- EA. Differential diagnosis of primary failure of eruption (PFE) with and without evidence of pathogenic mutations in the PTHR1 gene. J Orofac Orthop. 2014;75:226-39.

14. Frazier-Bowers S, Koeheler K, Ackerman J, Proffit W. Primary Failure of eruption: further characterization of a rare eruption disorder. Am J Orthod Dentofacial Orthop. 2007;131(578):e1-11.

15. Decker E, Stellzing-Eisenhauer A, Fiebig BS, Rau C, Kress W, Saar K, Ruschendorf F, Hubner N, Grimm T, Weber BH. PTH1R loss-of-function mutations in familial, nonsyndromic primary failure of tooth eruption. Am J Hum Genet. 2008;83:781-6.

16. Stellzig-Eisenhauer A, Decker E, Meyer-Marcotty P, Rau C, Fiebing BS, Kress W. Primary failure of eruption (PFE) — clinical and molecular genetics analysis. J Orofac Orthop. 2010;71:6-16.

17. Yamaguchi T, Hosomichi K, Narita A, Shirota T, Tomoyasu Y, Maki K, Inoue I. Exome resequencing combined with linkage analysis identifies novel PTH1R variants in primary failure of tooth eruption in Japanese. J Bone Miner Res. 2011;26:1655-61.

18. Roth H, Fritsche LG, Meier C, Pilz P, Eigenthaler M, Meyer-Marcotty P, Stellzig-Eisenhauer A, Proff P, Kanno CM, Weber BH. Expanding the spectrum of PTH1R mutations in patients with primary failure of tooth eruption. Clin Oral Investig. 2014;18:377-84.

19. Hanisch M, Hanisch L, Kleinheinz J, Jung S. Primary failure of eruption (PFE): a systematic review. Head Face Med. 2018;14:5.

20. Schipani E, Kruse K, Jüppner H. A constitutively active mutant PTHPTHrP receptor in Jansen type metaphyseal chondrodysplasia. Science. 1995;268:98-100.

21. Jobert AS, Zhang P, Couvineau A, Bonaventure J, Roume J, Le Merrer M, Silve C. Absence of functional receptors for parathyroid hormone and parathyroid hormone-related peptide in Blomstrand chondrodysplasia. J Clin Invest. 1998;102:34-40.

22. Duchatelet S, Ostergaard E, Cortes D, Lemainque A, Julier C. Recessive mutations in PTHR1 cause contrasting skeletal dysplasias in Eiken and Blomstrand syndromes. Hum Mol Genet. 2005;14:1-5.

23. Couvineau A, Wouters V, Bertrand G, Rouyer C, Gérard B, Boon LM, Grandchamp B, Vikkula M, Silve C. PTHR1 mutations associated with Ollier disease result in receptor loss of function. Hum Mol Genet. 2008;17:2766-75.

24. Frazier-Bowers S, Hendricks HM, Wight JL, Long K, Dibble CF, Bencharit S. Novel mutations in PTH1R associated with primary failure of eruption and osteoarthritis. J Dent Res. 2014;93:134-9.

25. Grippaudo C, Cafiero C, D’Apolito I, Ricci B, Frazier-Bowers SA. Primary failure of eruption: clinical and genetic findings in the mixed dentition. Angle Orthod. 2018;88:275-82.

26. Grippaudo C, Cafiero C, D'Apolito I, Re A, Genuardi M, Chiurazzi P, Frazier-Bowers SA. A novel nonsense PTH1R variant shows incomplete penetrance of primary failure of eruption: a case report. BMC Oral Health. 2019;19:249.

27. The Genome Aggregation Database. https://gnomad.broadinstitute.org/. Accessed 21 Jan 2021.

28. The Single Nucleotide Polymorphism Database (dbSNP). https://www. ncbi.nlm.nih.gov/snp. Accessed 22 Jan 2021

29. NCBI ClinVar database. https://www.ncbi.nlm.nih.gov/clinvar. Accessed 26 Jan 2021.

30. Leiden Open Variation database. http://databases.lovd.nl/shared/genes/ PTH1R. Accessed 17 Jan 2021.

31. VarSome. https://varsome.com/. Accessed 21 Jan 2021.

32. Kopanos C, Tsiolkas V, Kouris A, Chapple CE, Albarca Aguilera M, Meyer $\mathrm{R}$, Massouras A. VarSome: the human genomic variant search engine. Bioinformatics. 2019;35:1978-80. 
33. Subramanian H, Döring F, Kollert S, Rukoyatkina N, Sturm J, Gambaryan S, Stellzig-Eisenhauer A, Meyer-Marcotty P, Eigenthaler M, Wischmeyer E. PTH1R mutants found in patients with primary failure of tooth eruption disrupt G-protein signaling. PLoS ONE. 2016;11:e0167033.

34. Hendricks HM, Bencharit S, Seaman W, Frazier-Bowers SA. In silico and functional evaluation of PTH1R mutations found in patients with primary failure of eruption (PFE). Orthod Craniofac Res. 2017;20(Suppl. 1):57-62.

35. Izumida E, Suzawa T, Miyamoto Y, Yamada A, Otsu M, Saito T, Yamaguchi T, Nishimura K, Ohtaka M, Nakanishi M, Yoshimura K, Sasa K, Takimoto R, Uyama R, Shirota T, Maki K, Kamijo R. Functional analysis of PTH1R variants found in primary failure of eruption. J Dent Res. 2020;99:429-36.

36. Philbrick WM, Dreyer BE, Nachbandi IA, Karaplis AC. Parathyroid hormonerelated protein is required for tooth eruption. Proc Natl Acad Sci USA. 1998:95:11846-51.

37. Tokavanich N, Gupta A, Nagata M, Takahashi A, Matsushita Y, Yatabe M, Ruellas A, Cevidanes L, Maki K, Yamaguchi T, Ono N, Ono W. A threedimensional analysis of primary failure of eruption in humans and mice. Oral Dis. 2020;26:391-400

38. Nagata $\mathrm{M}, \mathrm{Ono} \mathrm{N}$, Ono W. Mesenchymal progenitor regulation of tooth eruption: a view from PTHrP. J Dent Res. 2020:99:133-42.

39. Klopocki E, Hennig BP, Dathe K, Koll R, de Ravel T, Baten E, Blom E, Gillerot Y, Weigel JF, Krüger G, Hiort O, Seemann P, Mundlos S. Deletion and point mutations of PTHLH cause brachydactyly type E. Am J Hum Genet. 2010;86:434-9.

\section{Publisher's Note}

Springer Nature remains neutral with regard to jurisdictional claims in published maps and institutional affiliations.

\section{Submit your manuscript to a SpringerOpen ${ }^{\circ}$ journal and benefit from:}

- Convenient online submission

- Rigorous peer review

- Open access: articles freely available online

- High visibility within the field

- Retaining the copyright to your article

Submit your next manuscript at $\boldsymbol{\nabla}$ springeropen.com 